\title{
Local truncation error of low-order fractional variational integrators ${ }^{\star}$
}

\author{
Fernando Jiménez and Sina Ober-Blöbaum \\ Department of Engineering Science, University of Oxford \\ Parks Road, Oxford OXI 3PJ, UK \\ fernando.jimenez@eng.ox.ac.uk, sina.ober-blobaum@eng.ox.ac.uk
}

\begin{abstract}
We study the local truncation error of the so-called fractional variational integrators, recently developed in [12] based on previous work by Riewe and Cresson [34]. These integrators are obtained through two main elements: the enlarging of the usual mechanical phase space by the introduction of the fractional derivatives of the dynamical curves; and a discrete restricted variational principle, in the spirit of discrete mechanics and variational integrators [5]. The fractional variational integrators are designed for modelling fractional dissipative systems, which, in particular cases, reduce to mechanical systems with linear damping. All these elements are introduced in the paper. In addition, as original result, we prove (\$3 Theorem 22 the order of local truncation error of the fractional variational integrators with respect to the dynamics of mechanical systems with linear damping.
\end{abstract}

Keywords: Fractional variational integrators · Dissipative systems · Local truncation error

\section{Preliminaries}

\subsection{Local truncation error}

Let $z:[a, b] \rightarrow \mathbb{R}^{d}$ and $f: \mathbb{R}^{d} \rightarrow \mathbb{R}^{d}$ a smooth curve and a smooth vector field, respectively, for $d \in \mathbb{N}$ and $[a, b] \subset \mathbb{R}$. Using the usual dot notation as time derivative we can define the initial value problem

$$
\dot{z}=f(z), \quad z(a)=z_{0},
$$

$z_{0} \in \mathbb{R}^{d}$, with smooth solution $z(t) \subset \mathbb{R}^{d}$. On the other hand, we define an implicit one-step numerical method:

$$
z_{k+1}=z_{k}+h f_{h}\left(z_{k}, z_{k+1}, h\right)
$$

where $h \in \mathbb{R}_{+}$is the time step, $f_{h}: \mathbb{R}^{d} \times \mathbb{R}^{d} \times \mathbb{R}_{+} \rightarrow \mathbb{R}^{d}$ is smooth, and $z_{k}$ is considered an approximation of $z\left(t_{k}\right)$ for the time grid $t_{k}=\{a+h k \mid k=0, \cdots, N\}$,

* This work has been funded by the EPSRC project: 'Fractional Variational Integration and Optimal Control"; ref: EP/P020402/1. 
with $N=(b-a) / h$. We say that the local truncation error order of the method (2) with respect to (1) is $p$ if

$$
\left\|z\left(t_{k+1}\right)-z_{k+1}\right\|=O\left(h^{p+1}\right),
$$

when $h \rightarrow 0$ and where $\|\cdot\|$ is the Euclidean norm in $\mathbb{R}^{d}[6]$.

\subsection{Conservative mechanical systems}

The dynamics of conservative simple mechanical systems, subject to a potential force [7, is described by the second-order differential equation:

$$
m \ddot{x}=-\nabla U(x), \quad x(a)=x_{0}, \dot{x}(a)=v_{0},
$$

where $x_{0}, v_{0} \in \mathbb{R}, m \in \mathbb{R}_{+}$is the mass of the system (for simplicity, we will set $m=1), x:[a, b] \rightarrow \mathbb{R}^{1}$ is the dynamical curve and the potential energy $U: \mathbb{R} \rightarrow \mathbb{R}$ is a smooth function. This equation can be transformed into a first-order differential equation:

$$
\begin{aligned}
& \dot{x}=v, \\
& \dot{v}=-\nabla U(x), \quad x(a)=x_{0}, \quad v(a)=v_{0} .
\end{aligned}
$$

The dynamical equation (4) can be obtained as a critical condition for extremals from the Hamilton's principle [8, given the action integral

$$
S(x)=\int_{a}^{b} L(x(t), \dot{x}(t)) d t
$$

for a Lagrangian function $L: T \mathbb{R} \rightarrow \mathbb{R}$ (we shall consider the tangent bundle $T \mathbb{R}$ as the space locally isomorphic to $\mathbb{R} \times \mathbb{R}$, with coordinates $(x, \dot{x}))$ defined by

$$
L(x, \dot{x})=\frac{1}{2} \dot{x}^{2}-U(x) .
$$

Remarkable geometric properties of the flow generated by (4) (equivalently (5)) are its symplecticity (it preserves the symplectic form $\Omega_{L}:=d x \wedge d \dot{x}=d x \wedge d v \in$ $\bigwedge^{2}(T \mathbb{R})$ ) and the preservation of symmetries (Noether's theorem) $[8]$.

Remark 1. Observe that we are choosing a "Lagrangian version" (5) of Hamilton equations for simple mechanical systems. In the picked setup, i.e. the configuration manifold is the real space and the particular Lagrangian function (7), both Lagrangian and Hamiltonian pictures are equivalent. Therefore, the theorems about the local truncation error order of variational integrators in [5]9], apply for (5).

\footnotetext{
${ }^{1}$ We will restrict to the real space $\mathbb{R}$ for sake of simplicity, but all results in these paper are straighforwardly extended to $\mathbb{R}^{d}$.
} 


\subsection{Variational integrators}

A natural way of obtaining integrators preserving the symplectic form $\Omega_{L}$ and the symmetries of the system is to construct variational integrators [5]. For that, we replace the continuous curves $x(t)$ by discrete ones $x_{d}=\left\{x_{k}\right\}_{0: N}:=$ $\left\{x_{0}, x_{1}, \cdots, x_{N}\right\} \in \mathbb{R}^{N+1}$. Moreover, we define the discrete Lagrangian $L_{d}$ : $\mathbb{R} \times \mathbb{R} \rightarrow \mathbb{R}$ as an approximation in one time step of the action integral (6), say

$$
L_{d}\left(x_{k}, x_{k+1}, h\right) \simeq \int_{t_{k}}^{t_{k}+h} L(x(t), \dot{x}(t)) d t,
$$

where we shall omit the $h$ dependence of the discrete Lagrangian unless needed. Given this, we define the discrete action sum $S_{d}\left(x_{d}\right)=\sum_{k=0}^{N-1} L_{d}\left(x_{k}, x_{k+1}\right)$; the discrete Hamilton's principle applied upon this action sum provides the so-called discrete Euler-Lagrange equations:

$$
D_{1} L_{d}\left(x_{k}, x_{k+1}\right)+D_{2} L_{d}\left(x_{k-1}, x_{k}\right)=0, \quad k=1, \ldots, N-1,
$$

which, under the condition $\left[D_{12} L_{d}\right]$ is regular, define a discrete flow $F_{L_{d}}: \mathbb{R} \times$ $\mathbb{R} \rightarrow \mathbb{R} \times \mathbb{R} ;\left(x_{k}, x_{k+1}\right) \mapsto\left(x_{k+1}, x_{k+2}\right)$, that we call variational integrator. Alternatively, the transformation 2 .

$$
\begin{aligned}
v_{k}^{-} & =-D_{1} L_{d}\left(x_{k}, x_{k+1}\right), \\
v_{k+1}^{+} & =D_{2} L_{d}\left(x_{k}, x_{k+1}\right),
\end{aligned}
$$

defines an alternate discrete flow $\tilde{F}_{L_{d}}: \mathbb{R} \times \mathbb{R} \rightarrow \mathbb{R} \times \mathbb{R} ;\left(x_{k}, v_{k}\right) \mapsto\left(x_{k+1}, v_{k+1}\right)$, which, when we pick the Lagrangian (7), will be a variational integrator for (5) (observe that, in the general case, the "velocity matching" condition $v_{k}^{-}=v_{k}^{+}$ reproduces the discrete Euler-Lagrange equations (9)). As mentioned above, $\tilde{F}_{L_{d}}$ is symplectic and momentum preserving. Moreover, the symplecticity ensures a bounded energy behaviour in the long-term, which is explained by Backward Error Analysis [6]. Another advantage of the variational approach is that the local truncation error order of the integrators can be determined from the approximation in (8). In particular, we can establish the following result, which is a direct application of the order theorems in [5] and [9]:

Theorem 1. Given the Lagrangian $L(x(t), \dot{x}(t))(7)$ and $L_{d}\left(x_{k}, x_{k+1}\right)$ an order $p$ approximation of the action integral (8), then the local truncation error of the variational integrator $\tilde{F}_{L_{d}}$ determined by 10 with respect to $(5)$ is of order $p$.

Low-order integrators (up to 2$)^{3}$ can be obtained through a first order quadrature and the following linerar interpolation between the points $\left[x_{k}, x_{k+1}\right]: \dot{x}\left(t_{k}\right) \simeq$ $\left(x_{k+1}-x_{k}\right) / h$ and $x\left(t_{k}\right) \simeq \gamma x_{k}+(1-\gamma) x_{k+1}$, where $\gamma \in[0,1] \subset \mathbb{R}$. Namely:

$$
L_{d}\left(x_{k}, x_{k+1}\right)=\frac{1}{2 h}\left(x_{k+1}-x_{k}\right)^{2}-h U\left(\gamma x_{k}+(1-\gamma) x_{k+1}\right) .
$$

\footnotetext{
${ }^{2}$ Naturally, this transformation is nothing but the discrete Legendre transform [5], which is shown here in a Lagrangian version.

${ }^{3}$ High-order variational integrators can be obtained via the use of inner discrete nodes and more involved interpolations, see [10].
} 
From this discrete Lagrangian, the discrete Euler-Lagrange equations read:

$\frac{x_{k+1}-2 x_{k}+x_{k-1}}{h^{2}}=-\gamma \nabla U\left(\gamma x_{k}+(1-\gamma) x_{k+1}\right)-(1-\gamma) \nabla U\left(\gamma x_{k-1}+(1-\gamma) x_{k}\right)$,

which are a discretization in finite differences of (4); whereas the flow $\tilde{F}_{L_{d}}$ defined by 10 reads:

$$
\begin{aligned}
& x_{k+1}=x_{k}+h v_{k}-h^{2} \gamma \nabla U\left(\gamma x_{k}+(1-\gamma) x_{k+1}\right), \\
& v_{k+1}=v_{k}-h \nabla U\left(\gamma x_{k}+(1-\gamma) x_{k+1}\right) .
\end{aligned}
$$

Using the Taylor expansion and the definition in $\$ 1.1$, it is easy to see that the order 2 of this integrator w.r.t. (5) is achieved when $\gamma=1 / 2$, i.e. for the midpoint rule, circumstance which is consistent with Theorem 1 .

\subsection{Linearly damped mechanical systems}

The dynamical equations of a mechanical system subject to linear damping are:

$$
\ddot{x}=-\nabla U(x)-\rho \dot{x}, \quad x(a)=x_{0}, \quad \dot{x}(a)=v_{0},
$$

with $\rho \in \mathbb{R}_{+}$. In the first-order version:

$$
\begin{aligned}
& \dot{x}=v, \\
& \dot{v}=-\nabla U(x)-\rho v, \quad x(a)=x_{0}, \quad v(a)=v_{0} .
\end{aligned}
$$

There is no Lagrangian function such that 12 are its Euler-Lagrange equations [11. With our fractional approach [1/2, explained in \$2, we have designed a restricted variational principle surpassing this issue.

\section{Fractional variational integrators}

\subsection{Continuous and discrete fractional derivatives}

Given a smooth function $g:[a, b] \rightarrow \mathbb{R}$, the $\alpha$-fractional derivatives (RiemannLiouville version), with $\alpha \in[0,1]$ are:

$D_{-}^{\alpha} g(t)=\frac{1}{\Gamma(1-\alpha)} \frac{d}{d t} \int_{a}^{t}(t-\tau)^{-\alpha} g(\tau) d \tau, D_{+}^{\alpha} g(t)=-\frac{1}{\Gamma(1-\alpha)} \frac{d}{d t} \int_{t}^{b}(\tau-t)^{-\alpha} g(\tau) d \tau$, where $\Gamma(z)$ is the Gamma function [12. Relevant properties in our approach are

$\int_{a}^{b} h(t) D_{\sigma}^{\alpha} g(t) d t=\int_{a}^{b}\left(D_{-\sigma}^{\alpha} h(t)\right) g(t) d t, D_{-}^{1 / 2} D_{-}^{1 / 2} g(t)=\dot{g}(t), \quad D_{+}^{1 / 2} D_{+}^{1 / 2} g(t)=-\dot{g}(t)$,

with $\sigma=\{-,+\}$. On the other hand, for a discrete curve $\left\{x_{k}\right\}_{0: N}$ and the time step $h \in \mathbb{R}_{+}$, we can define the following discrete $\alpha$-fractional derivatives [4]:

$$
\Delta_{-}^{\alpha} x_{k}:=\frac{1}{h^{\alpha}} \sum_{n=0}^{k} \alpha_{n} x_{k-n}, \quad \Delta_{+}^{\alpha} x_{k}:=\frac{1}{h^{\alpha}} \sum_{n=0}^{N-k} \alpha_{n} x_{k+n},
$$

where $\alpha_{n}:=-\alpha(1-\alpha)(2-\alpha) \cdots(n-1-\alpha) / n$ ! and $\alpha_{0}:=1$. It is proven in 13] (Theorem 2.4) that $\Delta_{ \pm}^{\alpha} x_{k}$ is an order 0 approximation (i.e. consistent) of $D_{ \pm}^{\alpha} x(t)$. 


\subsection{Continuous restricted variational principle}

In [1|2], the fractional phase space $\mathfrak{T R}$ is defined, which is a vector bundle over $\mathbb{R} \times \mathbb{R}$ with coordinates $\left(x, y, \dot{x}, \dot{y}, D_{-}^{\alpha} x, D_{+}^{\alpha} y\right)$ over the point $(x, y)$. This is an extension of the usual tangent bundle, including the fractional derivatives after doubling the space of curves (note that we are considering an extra curve $y(t)$ ). The necessity of this doubling comes out of the assymetric integration by parts rule in (14). Given this fractional phase space, we define a Lagrangian function $\mathcal{L}: \mathcal{T R} \rightarrow \mathbb{R}$ and the action integral:

$$
S((x, y))=\int_{a}^{b} \mathcal{L}\left(x(t), y(t), \dot{x}(t), \dot{y}(t), D_{-}^{\alpha} x(t), D_{+}^{\alpha} y(t)\right) d t .
$$

Using a particular set of varied curves $(x(t), y(t))_{\epsilon}:=(x(t), y(t))+\epsilon(\delta x(t), \delta x(t))$ (observe that we are "restricting" the variations of both curves to be equal), where $\epsilon \in \mathbb{R}_{+}$and $\delta x:[a, b] \rightarrow \mathbb{R}$ is smooth and defined such that $\delta x(a)=$ $\delta x(b)=0$, and considering the extremal condition of the action as $d /\left.d \epsilon\right|_{\epsilon=0} S\left((x, y)_{\epsilon}\right)=$ 0 , we obtain the next result.

Proposition 1. Given the Lagrangian function

$$
\mathcal{L}\left(x, y, \dot{x}, \dot{y}, D_{-}^{\alpha} x, D_{+}^{\alpha} y\right)=\left(\frac{1}{2} \dot{x}^{2}-U(x)\right)+\left(\frac{1}{2} \dot{y}^{2}-U(y)\right)-\rho D_{-}^{\alpha} x D_{+}^{\alpha} y,
$$

then, a sufficient condition for the extremals of (16) subject to restricted variations $(x, y)_{\epsilon}$ are the equations:

$$
\begin{aligned}
& \ddot{x}=-\nabla U(x)-\rho D_{-}^{\alpha} D_{-}^{\alpha} x \rightarrow(\alpha=1 / 2) \rightarrow \ddot{x}=-\nabla U(x)-\rho \dot{x}, \\
& \ddot{y}=-\nabla U(y)-\rho D_{+}^{\alpha} D_{+}^{\alpha} y \rightarrow(\alpha=1 / 2) \rightarrow \ddot{y}=-\nabla U(y)+\rho \dot{y} .
\end{aligned}
$$

The previous equations are the so-called restricted fractional Euler-Lagrange equations in [12] (see these references for the proof) for the particular Lagrangian (17). It can be rigourosly proven that the $y$-system is nothing but the $x$-system in reversed time (even for more general Lagrangians), and therefore these equations do not imply extra physics. For a general $\alpha$ we obtain the equations of a mechanical system subject to fractional damping. When $\alpha \rightarrow 1 / 2$, according to (14), we recover the dynamics of systems with linear damping (12).

\subsection{Discrete restricted variational principle}

Given discrete sequences $x_{d}=\left\{x_{k}\right\}_{0: N}$ and $y_{d}=\left\{y_{k}\right\}_{0: N}$ and defining $\dot{x}_{k}:=$ $\left(x_{k+1}-x_{k}\right) / h$; equiv. $y_{k} ; x_{k+1 / 2}:=\left(x_{k+1}+x_{k}\right) / 2$ and $x_{k-1 / 2}:=\left(x_{k}+x_{k-1}\right) / 2$; equiv. $y_{k \pm 1 / 2}$; (we pick the midpoint rule because it provides the maximum order of (11) w.r.t. (5)), the discrete action sum for the fractional problem is

$$
S_{d}\left(\left(x_{d}, y_{d}\right)\right)=\sum_{k=0}^{N-1} h \mathcal{L}\left(x_{k+1 / 2}, y_{k+1 / 2}, \dot{x}_{k}, \dot{y}_{k}, \Delta_{-}^{\alpha} x_{k}, \Delta_{+}^{\alpha} y_{k}\right),
$$


where the discrete fractional derivatives are defined in (15). As in the continuous case, we pick a particular set of restricted discrete variations, namely $\left(x_{d}, y_{d}\right)_{\epsilon}:=\left(\left\{x_{k}\right\}_{0: N},\left\{y_{k}\right\}_{0: N}\right)+\epsilon\left(\left\{\delta x_{k}\right\}_{0: N},\left\{\delta x_{k}\right\}_{0: N}\right)$, where $\left\{\delta x_{k}\right\}_{0: N}$ is such that $\delta x_{0}=\delta x_{N}=0$. Considering the extremal condition of the discrete action as $d /\left.d \epsilon\right|_{\epsilon=0} S_{d}\left(\left(x_{d}, y_{d}\right)_{\epsilon}\right)=0$, we get the next result:

Proposition 2. Given the Lagrangian $\mathcal{L}(17)$, a sufficient condition for the extremals of 119 subject to restricted variations $\left(x_{d}, y_{d}\right)_{\epsilon}$ is

$$
\begin{aligned}
\frac{x_{k+1}-2 x_{k}+x_{k}}{h^{2}} & =-\frac{1}{2} \nabla U\left(x_{k+1 / 2}\right)-\frac{1}{2} \nabla U\left(x_{k-1 / 2}\right)-\rho \Delta_{-}^{\alpha} \Delta_{-}^{\alpha} x_{k}, \\
\frac{y_{k+1}-2 y_{k}+y_{k}}{h^{2}} & =-\frac{1}{2} \nabla U\left(y_{k+1 / 2}\right)-\frac{1}{2} \nabla U\left(y_{k-1 / 2}\right)-\rho \Delta_{+}^{\alpha} \Delta_{+}^{\alpha} y_{k},
\end{aligned}
$$

for $k=1, \cdots, N-1$.

The previous equations are the so-called discrete restricted fractional EulerLagrange equations in 112 for the particular Lagrangian (17). In (20) we recognize a discretization in finite differences of 18 for a general $\alpha$. Moreover, it can be also rigourosly proven that the discrete $y$-system is the discrete $x$ system in reversed (discrete) time.

\section{Order result}

As original result, we explore the local truncation error order of 20 with respect to 13 . With that aim, we need to establish an equivalent to 10 in the fractional case. Based on [12], we pick (restricting to the $x$-system):

$$
\begin{aligned}
v_{k}^{-} & =\frac{x_{k+1}-x_{k}}{h}+\frac{h}{2} \nabla U\left(x_{k+1 / 2}\right)+h \rho \Delta_{-}^{\alpha} \Delta_{-}^{\alpha} x_{k}, \\
v_{k+1}^{+} & =\frac{x_{k+1}-x_{k}}{h}-\frac{h}{2} \nabla U\left(x_{k+1 / 2}\right) .
\end{aligned}
$$

Note that the first two terms in the right hand side of both equations corresponds to 10 for $L_{d}\left(x_{k}, x_{k+1}\right)=\left(x_{k+1}-x_{k}\right) / 2 h-h U\left(x_{k+1 / 2}\right)$. In addition, observe that the "velocity matching" condition $v_{k}^{-}=v_{k}^{+}$reproduces the discrete dynamics 20. Finally, according to 15 it can be proven [12] that

$$
\Delta_{-}^{1 / 2} \Delta_{-}^{1 / 2} x_{k}=\left(x_{k}-x_{k-1}\right) / h, \quad k=1, \cdots, N .
$$

With these elements, we can establish the following order result

Theorem 2. The local truncation order of the fractional variational integrators for simple mechanical systems (21) when $\alpha=1 / 2$, with respect to the continuous dynamics 13), is one. 
Proof. First, using Taylor expansions and setting the notation $x\left(t_{k}\right):=x_{k}, \dot{x}\left(t_{k}\right):=$ $v_{k}, v\left(t_{k}\right):=v_{k}$, we deliver expressions for $x\left(t_{k+1}\right)$ and $v\left(t_{k+1}\right)$ in terms of the dynamics 13 , namely:

$$
\begin{aligned}
& x\left(t_{k}+h\right)=x_{k}+h v_{k}-\frac{h^{2}}{2} \nabla U\left(x_{k}\right)-\frac{h^{2}}{2} \rho v_{k}+O\left(h^{3}\right), \\
& v\left(t_{k}+h\right)=v_{k}-h \nabla U\left(x_{k}\right)-h \rho v_{k}-\frac{h^{2}}{2} \Delta U\left(x_{k}\right) v_{k} \\
&+\frac{h^{2}}{2} \rho \nabla U\left(x_{k}\right)+\frac{h^{2}}{2} \rho^{2} v_{k}+O\left(h^{3}\right) .
\end{aligned}
$$

On the other hand, from 21) we get the integrator:

$$
\begin{aligned}
& x_{k+1}=x_{k}+h v_{k+1}+\frac{h^{2}}{2} \nabla U\left(x_{k+1 / 2}\right), \\
& v_{k+1}=v_{k}-h \nabla U\left(x_{k+1 / 2}\right)-h \rho \Delta_{-}^{1 / 2} \Delta_{-}^{1 / 2} x_{k} .
\end{aligned}
$$

Replacing 24b into 24a) we get

$$
\begin{aligned}
x_{k+1} & =x_{k}+h v_{k}-\frac{h^{2}}{2} \nabla U\left(x_{k+1 / 2}\right)-h^{2} \rho \Delta_{-}^{1 / 2} \Delta_{-}^{1 / 2} x_{k} \\
& ={ }^{1} x_{k}+h v_{k}-\frac{h^{2}}{2} \nabla U\left(x_{k+1 / 2}\right)-h^{2} \rho\left(\frac{x_{k}-x_{k-1}}{h}\right) \\
& ={ }^{2} x_{k}+h v_{k}-\frac{h^{2}}{2} \nabla U\left(x_{k}\right)-h^{2} \rho v_{k}+O\left(h^{3}\right),
\end{aligned}
$$

where in $=^{1}$ we have used 22 and in $=^{2}$ we have used $x_{k+1 / 2}=x_{k}+h v_{k} / 2+$ $O\left(h^{2}\right)$ and $\left(x_{k}-x_{k-1}\right) / h=v_{k}+h \nabla U\left(x_{k-1 / 2}\right) / 2$, according to 21b). Thus, from the last expression and (23a), it follows that $\left\|x\left(t_{k+1}\right)-x_{k+1}\right\|=O\left(h^{2}\right)$. From (24b) we get

$$
\begin{aligned}
v_{k+1} & =v_{k}-h \nabla U\left(x_{k+1 / 2}\right)-h \rho \Delta_{-}^{1 / 2} \Delta_{-}^{1 / 2} x_{k}=v_{k}-h \nabla U\left(x_{k+1 / 2}\right)-h \rho\left(\frac{x_{k}-x_{k-1}}{h}\right) \\
& =v_{k}-h \nabla U\left(x_{k}\right)-h \rho v_{k}-\frac{h^{2}}{2} \Delta U\left(x_{k}\right) v_{k}-\frac{h^{2}}{2} \rho \nabla U\left(x_{k-1 / 2}\right)+O\left(h^{3}\right) \\
& =v_{k}-h \nabla U\left(x_{k}\right)-h \rho v_{k}-\frac{h^{2}}{2} \Delta U\left(x_{k}\right) v_{k}-\frac{h^{2}}{2} \rho \nabla U\left(x_{k}\right)+O\left(h^{3}\right),
\end{aligned}
$$

where we have taken into account that $x_{k-1 / 2}=x_{k}+O(h)$ and used the same expressions as above for $x_{k+1 / 2}$ and $\left(x_{k}-x_{k-1}\right) / h$. From this last expression and (23b), we obtain that $\left\|v\left(t_{k+1}\right)-v_{k+1}\right\|=O\left(h^{2}\right)$, and the result follows from the definition of local truncation error in $\$ 1.1$.

Remark 2. The alternate integrator:

$v_{k}^{-}=\frac{x_{k+1}-x_{k}}{h}+\frac{h}{2} \nabla U\left(x_{k+1 / 2}\right), \quad v_{k+1}^{+}=\frac{x_{k+1}-x_{k}}{h}-\frac{h}{2} \nabla U\left(x_{k+1 / 2}\right)-h \rho \Delta_{-}^{\alpha} \Delta_{-}^{\alpha} x_{k+1}$,

which also reproduces 20 via velocity matching $v_{k}^{-}=v_{k}^{+}$, delivers the same result. 


\section{Conclusions}

We prove that the local truncation error order of the fractional variational integrators (21), with respect to the dynamics of linearly damped mechanical systems (13), is one. These integrators are designed in the spirit of variational integrators [5], i.e. by means of the discretization of variational principles, in our case the Hamilton's principle with restricted variations. Thus, we expect similar behaviour in terms of Theorem 1 i.e. the order of the approximation of the action is equal to the order of the integrator. Our result is not coherent in the fractional case. On the one hand, we pick the midpoint rule approximation $x\left(t_{k}\right) \simeq\left(x_{k}+x_{k+1}\right) / 2$, which is the case where the maximum order (2) is achieved for the usual variational integrators and conservative mechanical systems. On the other, the approximation of the fractional derivative that we use, $\Delta_{-}^{\alpha} x_{k}$, is only consistent (order 0$)$ w.r.t. $D_{-}^{\alpha} x(t)([13$, Theorem 2.4). Thus, the approximation of the action (16) is limited to $O(h)$, whereas the integrator is $O\left(h^{2}\right)$. This represents an improvement from the expected result.

\section{References}

1. Jiménez, F. and Ober-Blöbaum, S.: A fractional variational approach for modelling dissipative mechanical systems: continuous and discrete settings. 6th IFAC LHMNC2018 Proceedings, IFAC-PapersOnLine, 51(3), 50-55, (2018)

2. Jiménez, F. and Ober-Blöbaum, S.: Fractional damping through restricted calculus of variations. In preparation, (2019)

3. Riewe, F.: Nonconservative Lagrangian and Hamiltonian mechanics. Phys. Rev. E 53(2), 1890-1899, (1996)

4. Cresson, J. and Inizan, P.: Variational formulations of differential equations and asymmetric fractional embedding. Journal of Mathematical Analysis and Applications, 385(2), 975-997, (2012)

5. Marsden, J.E. and West, M.: Discrete mechanics and variational integrators. Acta Numerica (10), 357-514, (2001)

6. Hairer, E., Lubich, C. and Wanner, G.: Geometric Numerical Integration, StructurePreserving Algorithms for Ordinary Differential Equations. Springer Series in Computational Mathematics, 31, Springer-Verlag Berlin, (2002)

7. Goldstein, H., Poole, C. and Safko, J.: Classical Mechanics. 3rd edn. Addison Wesley, (2001)

8. Abraham, R. and Marsden, J.E.: Foundations of Mechanics. Benjamin-Cummings Publ. Co., (1978)

9. Patrick, C.W. and Cuell, C.: Error analysis of variational integrators of unconstrained Lagrangian systems. Numer. Math. (113)(2), 243-264, (2009)

10. Ober-Blöbaum, S. and Saake, N.: Construction and analysis of higher order Galerkin variational integrators. Adv. Comput. Math. 41(6), 955986, (2015)

11. Bauer, P.S.: Dissipative dynamical systems. Proc. Nat. Acad. Sci. 17, 311-314, (1931)

12. Samko, S., Kilbas, A. and Marichev, O.: Fractional integrals and derivatives: theory and applications. Gordon and Breach, Yverdon, (1993)

13. Meerschaert, M.M. and Tadjeran, Ch.: Finite difference approximations for fractional advection-dispersion flow equations. J. of Comp. and Appl. Math. 172, 65-77, (2004) 